\title{
Neutrophilic dermatosis of the dorsal hands: A new case report associated with Basedow's disease
}

\section{Aida Oulehri', Sara Elloudi', Hanane Baybay', Zakia Douhi', Mouna Rimani², Fatima Zahra Mernissi ${ }^{1}$}

\author{
${ }^{1}$ Department of Dermatology, University Hospital Hassan II, Fez, Morocco, ${ }^{2}$ Hassan Center of Anatomy and Pathological \\ Cytology Laboratory, Rabat, Morroco
}

Corresponding author: Aida Oulehri, MD, E-mail: aidaoulehri@gmail.com

\begin{abstract}
Neutrophilic dermatosis of the dorsal hands (NDDH) is a newly described and poorly known disease, a topographic variant of Sweet's syndrome, most often clinically misdiagnosed as an infectious process, which, as a result, delays treatment. In addition, its association with underlying systemic and neoplastic disorders increases the need for accurate and early diagnosis. Interestingly, our case had an additional lesion located on one leg. Our patient also presented bacterial conjunctivitis, which may have either been part of the clinical presentation or the trigger of this entity and which was an association with Basedow's disease never described before.
\end{abstract}

Key words: Neutrophilic dermatosis; Dorsal hands; Basedow's disease

\section{INTRODUCTION}

Sweet's syndrome, originally described by Dr. Robert Douglas Sweet in 1964, is characterized by an acute onset of fever, leukocytosis, and tender erythematous plaques infiltrated by mature neutrophils. The lesions usually involve the face and extremities and show an excellent response to corticosteroids [1]. Recently, several new variants have been described, including neutrophilic dermatosis of the dorsal hands (NDDH). NDDH is characterized by tender erythematous plaques, pustules, bullae, and/or ulcers usually limited to the dorsa of the hands. Both Sweet's syndrome and its dorsal hand variant have been reported in association with malignancies, inflammatory bowel diseases, and drugs [2]. We report a case associated with Basedow's disease in a 61-year-old female.

\section{CASE REPORT}

A 61-year-old female was admitted to our hospital with a painful eruption on the hands. She had been followed since ten years of age for Basedow's disease with exophthalmos, for which she had been taking carbimazole, and had, besides, no notable medical history. Five days prior to the consultation, the patient presented herself with flu-like syndrome manifesting fever, arthralgia, myalgia, rhinorrhea, and pharyngitis, for which the patient took emergency-prescribed antibiotics and paracetamol. Subsequently, the patient developed redness of the eyes observed by an ophthalmologist who diagnosed bacterial conjunctivitis without further eye damage and treated it locally. Several days later, the patient felt painful tension in both hands, which gradually swelled. A physical examination revealed tense erythematous edema of both hands with the presence of multiple deep-seated pustules and purplish papules on the backs of the hands (Fig. 1). An examination of the rest of the skin found one purplish patch on the right leg (Fig. 2). The rest of the examination was normal, except for exophthalmos and bacterial conjunctivitis (Fig. 3). Laboratory tests on admission showed a total leukocyte count of $12.4 \times 109 / \mathrm{L}$ with $75 \%$ neutrophils. C-reactive protein was $50 \mathrm{mg} / \mathrm{L}$ (0-6 mg/L). Urine analysis, serum electrolytes, and

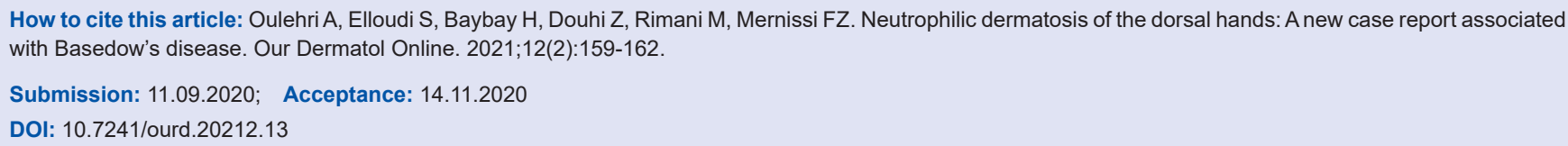




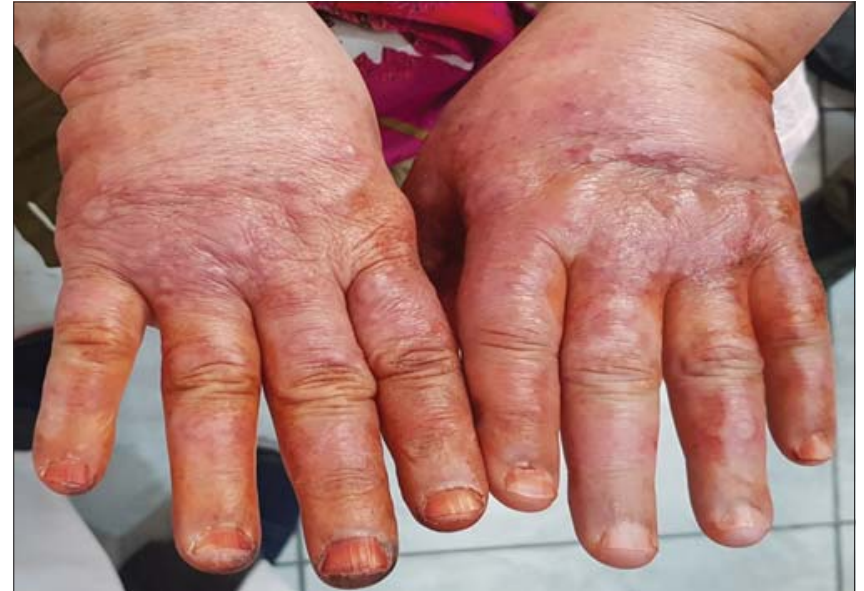

Figure 1: Tense and painful edema with multiple deep-seated pustules and purplish papules on the back of the hands (exogenous orange coloration due to henna).

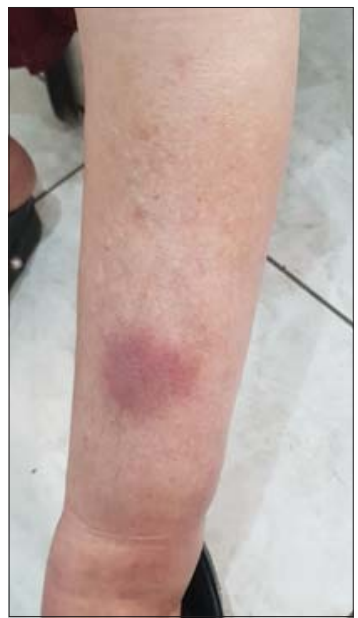

Figure 2: An additional isolated purplish patch on the right leg.

liver and renal function were normal. Electrophoresed proteins had an inflammatory profile. No abnormality was detected on a chest X-ray. A histopathologic examination revealed a dense dermal infiltrate of neutrophils restricted to the papillary and superficial reticular dermis with associated dermal edema (Fig. 4). There was no leukocytoclastic vasculitis. In the light of the patient's history, the physical examination, and the histopathologic features found, the diagnosis of neutrophilic dermatosis of the dorsal hands (NDDH) was reached. Treatment with indomethacin at an initial daily dose of $150 \mathrm{mg}$ for one week, and reduced to $100 \mathrm{mg}$ daily for the next two weeks, was initiated with remarkable improvement observed over the next week. The bacterial conjunctivitis completely regressed under the treatment. Complete resolution of all lesions was observed over the next two weeks. No recurrence was noted six months after the initial episode.

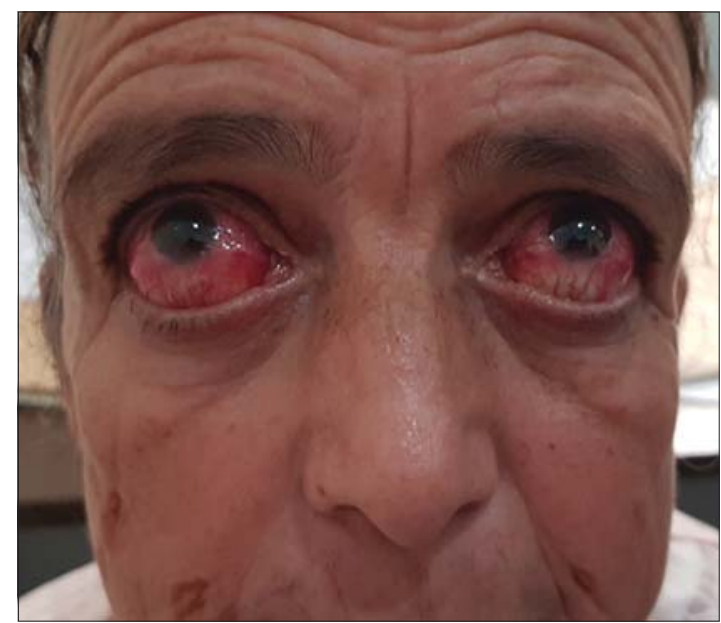

Figure 3: Chronic exophthalmos with bacterial conjunctivitis.

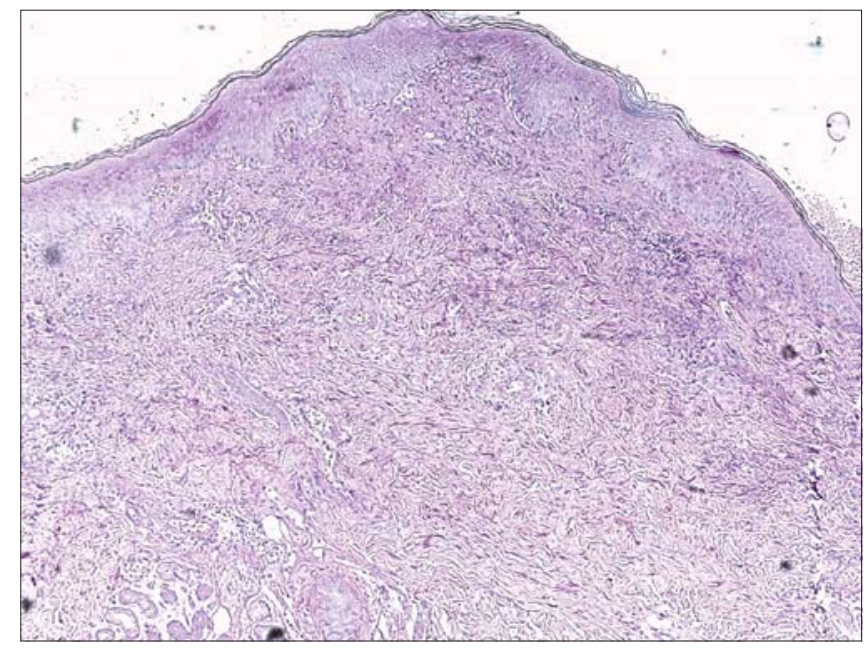

Figure 4: A dense dermal infiltrate of neutrophils restricted to the papillary and superficial reticular dermis with associated dermal edema.

\section{DISCUSSION}

Pustular vasculitis of the hands was first described by Strutton et al. in 1995 as a rash on the dorsal hands resembling Sweet's syndrome with histological leukocytoclastic vasculitis [3]. Subsequently, clinically similar cases were reported but without vasculitis $[4,5]$. Neutrophil dermatosis of the dorsal hands (NDDH), a term proposed by Galaria et al. in 2000, is now a widely accepted name for this clinicopathological condition [6].

Today, NDDH most commonly represents a "distributional or localized variant" of Sweet's syndrome, which belongs to the spectrum of neutrophilic dermatoses, rather than a primary vasculitis, with any vasculitis observed histologically being a secondary phenomenon. The different timings of the biopsies 
taken during the evolutionary phases of the lesions are likely to result in different findings with regard to the presence or absence of vasculitis [7].

The number of report cases of this entity remains limited [8-11]. A total of 123 cases were collected in a recent literature review [2].

Epidemiologically, there is a slight predominance in females, with a mean age of occurrence of 62.1 years [2], which is in accordance with our patient. These statistics are significantly different compared to those of Sweet's syndrome, which shows a female-to-male ratio of 4:1 and tends to occur in middle-aged females, often aged 30 to 50 years [12].

Clinically, the lesions are very similar to classic Sweet's syndrome with painful erythematous and purplish papules and plaques. Nodules, pustules, and hemorrhagic bullae that become ulcerated have also been described. The most common sites of eruption are the dorsal surfaces of both hands. The backs of the fingers or wrists may also be affected, with rare involvement of the palms. Both hands were affected in the majority of cases but unilateral involvement was also described and in $30.9 \%$ of cases [2]. There may be simultaneous or subsequent lesions elsewhere, such as on the back, lips, legs, and forehead $[9,11,13]$, as in our patient, who developed an isolated lesion on the right leg. The most common clinical differential diagnosis is an infection, for which the patient receives antibiotics but with no success.

Pathologically, NDDH is considered a variant of Sweet's syndrome with identical histological findings. Histologically, there is prominent papillary dermal edema, superficial and deep perivascular, and diffuse infiltrate of neutrophils with leukocytoclasia, extravasated erythrocytes, and no vasculitis. Admixed lymphocytes and occasional eosinophils may also be seen. It appears that any vasculitis observed is of secondary type rather than a primary phenomenon, similarly to PG or Sweet's syndrome $[7,11]$. Is usually only identified in early lesions and this phenomenon most likely represents a reaction to endothelial damage [14].

Hematological and serological investigations may, in NDDH, reveal neutrophilic leukocytosis and raised levels of CRP, although these are less frequently identified than in classical Sweet's syndrome and in keeping with a more localized variant of the disease [2]. Indeed, our patient manifested slight hyperleukocytosis at PNN and a moderate CRP level at 50.

Associations have been reported with a variety of diseases, while the majority of the comorbid conditions were diagnosed prior to the NDDH; several were diagnosed concomitantly to or shortly after a diagnosis of NDDH [2]. The most common are hematologic disorders, such as myelodysplastic syndrome, acute leukemia, and lymphoma $[11,15]$. In addition, solid neoplasms may be seen, such as cancers of the breast, kidney, colon, stomach, lung, and hypopharynx. Associated non-neoplastic disorders include inflammatory bowel diseases such as ulcerative colitis and Crohn's disease [7] and recent infections, by far the most common of which have been respiratory tract infections $[2,7,11]$. According to our knowledge, this is the first case associated with a thyroid pathology such as Basedow's disease. These findings highlight the importance of a thorough systematic inquiry in patients with NDDH. This may reveal a history of recent infection or guide the clinician to further investigation of inflammatory bowel diseases or solid organ tumors. Given the fact that hematological disorders have been reported in almost $15 \%$ of cases, a full blood count and serum protein electrophoresis would be sensible in all patients [2].

Multiple therapies have been used for NDDH with different rates of success and relapse. The treatment options for NDDH are similar to those for Sweet's syndrome. Corticosteroids are commonly used as firstline therapy; the majority of cases usually respond well to moderate doses of systemic corticosteroids. Dapsone has also been used either alone or in combination with systemic corticosteroids [13].

Several alternative therapies have been proposed, including cyclosporine, methotrexate, and Salazopyrin. Successful treatment with indomethacin, intravenous immunoglobulin, colchicine, minocycline, azathioprine, intralesional triamcinolone, topical steroids, and topical tacrolimus has also been reported $[16,17]$.

According to the latest review on the subject [2], and given that all treatment modalities were successful in yielding improvement within several weeks, we suggest it to be worth considering topical steroid monotherapy or non-steroid systemic agents guided by the patient's comorbidities and by the side-effect 
profile of the respective agent. Indeed, a topical steroid and/or an oral steroid-sparing agent appear to be as effective as systemic corticosteroids and may have a better side-effect profile. This was the same approach that we used with our patient, who received indometacin alone with complete remission after two weeks and no relapse. The current follow-up is at six months.

\section{CONCLUSION}

Although NDDH is an infrequent entity, the increase in cases reported in the literature proves that NDDH is not as rare as it may seem and that it is likely to be underdiagnosed, as it is mostly unrecognized.

A correct diagnosis would avoid unnecessary antibiotic treatments and potentially aggressive management strategies, such as surgical debridement, and will prompt search to exclude any possible association, particularly hematological malignancy, but also an occult solid organ, an inflammatory bowel disease, or infection, based on a history and examination.

\section{Consent}

The examination of the patient was conducted according to the principles of the Declaration of Helsinki.

The authors certify that they have obtained all appropriate patient consent forms, in which the patients gave their consent for images and other clinical information to be included in the journal. The patients understand that their names and initials will not be published and due effort will be made to conceal their identity, but that anonymity cannot be guaranteed.

\section{REFERENCES}

1. Kaur S, Gupta D, Garg B, Sood N. Neutrophilic dermatosis of dorsal hands. Indian Dermatol Online J. 2015;6:42.

2. Micallef D, Bonnici M, Pisani D, Boffa MJ. Neutrophilic Dermatosis of the Dorsal Hands: A Review of 123 Cases. J Am Acad Dermatol. 2019;S0190-9622:32678-7.

3. Struton G, Weedon D, Robertson I. Pustular vasculitis of the hands. J Am Acad Dermatol. 1995;32:192-198.

4. Nofal A, Abdelmaksoud A, Amer H, Nofal E, Yosef A, Gharib K, et al. Sweet's syndrome: diagnostic criteria revisited. J Dtsch Dermatol Ges. 2017;15:1081-8.

5. Filosa A, Filosa G. Neutrophilic dermatoses: a broad spectrum of disease. G Ital Dermatol Venereol. 2018;153:265-72.

6. Galaria NA, Junkins-Hopkins JM, Kligman D, James WD. Neutrophilic dermatosis of the dorsal hands: pustular vasculitis revisited. J Am Acad Dermatol. 2000;43:870-4.

7. Mobini N, Sadrolashrafi K, Michaels S. Neutrophilic Dermatosis of the Dorsal Hands: Report of a case and review of the literature. Case Rep Dermatol Med. 2019;2019:8301585.

8. Selvakumar Monisha D, Narasappa Raghavendra B, Kumar Patra A. A case of infantile Sweet's syndrome. Our Dermatol Online. 2020;11:143-5.

9. Fustà-Novell X, Bermejo S, Creus-Vila L. Neutrophilic dermatosis of the dorsal hands. Dermatosis neutrofílica del dorso de manos. Rev Clin Esp. 2020;S0014-2565:30056-4.

10. Zeraiq L, Lindahl KH, Bygum A. Sweet syndrome manifestations. Ugeskr Laeger. 2018;180:V10170786.

11. Cheng AMY, Cheng HS, Smith BJ, Stewart DA. Neutrophilic dermatosis of the hands: a review of 17 cases. J Hand Surg Am. 2018;43:185.e1-185.e5.

12. Villarreal-Villarreal CD, Ocampo-Candiani J, Villarreal-Martínez A. Sweet syndrome: a review and update. Actas Dermosifiliogr. 2016;107:369-78.

13. Behrangi E, Rasi A, Attar B, Azizian Z. Neutrophilic dermatosis of dorsal hands and legs. Arch Iran Med. 2016;19:879-81.

14. Rochet NM, Chavan RN, Cappel MA, Wada DA, Gibson LE. Sweet syndrome: clinical presentation, associations, and response to treatment in 77 patients. J Am Acad Dermatol. 2013;69:557-64

15. Leecy T, Anderson A, Von Nida J, Harvey N, Wood B. Neutrophilic dermatosis of the dorsal hands: an often under recognised and mistreated entity. Pathology. 2013;45:198-200.

16. Peteln I, Dolenc-Voljč M, Jurčić V. Neutrophilic dermatosis of the dorsal hands (acral Sweet syndrome) with predominantly lymphocytic dermal infiltrate. J Cutan Pathol. 2020;47:104-7.

17. Ramos FS, Ferreira FR, Rabay FMO, Lira MLA. Neutrophilic dermatosis of the dorsal hands: response to dapsone monotherapy. An Bras Dermatol. 2018;93:730-2.

Copyright by Aida Oulehri, et al. This is an open-access article distributed under the terms of the Creative Commons Attribution License, which permits unrestricted use, distribution, and reproduction in any medium, provided the original author and source are credited.

Source of Support: Nil, Conflict of Interest: None declared. 